OPEN ACCESS

Edited by:

Jonathan Shaw,

University of Sheffield, UK

Reviewed by:

Marquita Vernescia Gittens-St. Hilaire,

University of the West Indies,

Barbados

Jesús Santos,

University of León, Spain

*Correspondence:

Maria J. Figueras

mariajose.figueras@urv.cat

Peter Teunis

peter.teunis@rivm.n

Specialty section:

This article was submitted to

Infectious Diseases,

a section of the journal

Frontiers in Microbiology

Received: 27 June 2016

Accepted: 23 August 2016 Published: 21 September 2016

Citation:

Teunis $P$ and Figueras MJ (2016)

Reassessment of the

Enteropathogenicity of Mesophilic

Aeromonas Species.

Front. Microbiol. 7:1395.

doi: 10.3389/fmicb.2016.01395

\section{Reassessment of the Enteropathogenicity of Mesophilic Aeromonas Species}

\author{
Peter Teunis ${ }^{1,2 *}$ and Maria J. Figueras ${ }^{3 *}$ \\ ${ }^{1}$ Centre for Zoonoses and Environmental Microbiology, Centre for Infectious Disease Control, National Institute for Public \\ Health and the Environment, Bilthoven, Netherlands, ${ }^{2}$ Center for Global Safe WASH, Hubert Department of Global Health, \\ Rollins School of Public Health, Emory University, Atlanta, GA, USA, ${ }^{3}$ Unitat de Microbiologia, Departament de Ciènces \\ Médiques Bàsiques, Facultat de Medicina i Ciències de la Salut, Pere Virgili Institute for Health Research, Universitat Rovira i \\ Virgili, Reus, Spain
}

Cases of Aeromonas diarrhea have been described all over the world. The genus Aeromonas includes ca. 30 species, of which 10 have been isolated in association with gastroenteritis. The dominating species that account for ca. $96 \%$ of the identified strains are Aeromonas caviae, A. veronii, $A$. dhakensis, and A. hydrophila. However, the role of Aeromonas as a true enteropathogen has been questioned on the basis of the lack of outbreaks, the non-fulfillment of Koch's postulates and the low numbers of acute illnesses in the only existing human challenge study. In the present study we reassess the enteropathogenicity of Aeromonas using dose response models for microbial infection and acute illness. The analysis uses the data from the human challenge study and additional data from selected outbreak investigations where the numbers exposed and the dose were reported, allowing their inclusion as "natural experiments". In the challenge study several cases of asymptomatic shedding were found $(26.3 \%, 15 / 57)$, however, only $3.5 \%(2 / 57)$ of those challenged with Aeromonas developed acute enteric symptoms (i.e., diarrhea). The "natural experiments" showed a much higher risk of illness associated with exposure to Aeromonas, even at moderate to low doses. The median dose required for $1 \%$ illness risk, was $\sim 1.4 \times 10^{4}$ times higher in the challenge study $\left(1.24 \times 10^{4} \mathrm{cfu}\right)$ compared to natural exposure events (0.9 cfu). The dose response assessment presented in this study shows that the combined challenge and outbreak data are consistent with high infectivity of Aeromonas, and a wide range of susceptibility to acute enteric illness. To illustrate the outcomes, we simulate the risk associated with concentrations of Aeromonas found in different water and food matrices, indicating the disease burden potentially associated with these bacteria. In conclusion this study showed that Aeromonas is highly infectious, and that human susceptibility to illness may be high, similar to undisputed enteropathogens like Campylobacter or Salmonella.

Keywords: Aeromonas, outbreaks, water, food, challenge study, infective dose, risk of infection, risk of illness 


\section{INTRODUCTION}

The genus Aeromonas, includes Gram-negative, non-sporeforming rods that are autochthonous of the aquatic environments worldwide (Austin et al., 1996; Janda and Abbott, 2010; Figueras and Beaz-Hidalgo, 2015; Graf, 2015). In humans diarrhea, and wound infections are the most common presentation followed by bacteremia (Figueras, 2005; von Graevenitz, 2007; Janda and Abbott, 2010; Figueras and Beaz-Hidalgo, 2015). Isolation of Aeromonas in patients with diarrhea varies from ca 2 to $10 \%$ but a higher incidence (13\%) has been found in children from Nigeria (Figueras and Beaz-Hidalgo, 2015). Also a significantly higher incidence of Aeromonas is found in patients with diarrhea than in those considered asymptomatic carriers (Figueras, 2005; Janda and Abbott, 2010; Figueras and Beaz-Hidalgo, 2015). Typical susceptible patients in the different studies include babies, young children and the elderly and especially those with a preexisting illness and/or immunocompromised (Janda and Abbott, 2010; Figueras and Beaz-Hidalgo, 2015). According to Janda and Abbott (2010) it is important to report the presence of Aeromonas in stools of immunocompromised patients even if they are only asymptomatic because the risk of invasion and dissemination is considered inherently high for these patients. Nevertheless the role of Aeromonas in gastroenteritis has been questioned (Janda and Abbott, 1998, 2010; Chu et al., 2006; von Graevenitz, 2007), mainly because 55 out 57 challenged volunteers with a high dose of Aeromonas did not developed any symptoms of enteric illness, and due to the few reported outbreaks (Morgan et al., 1985; Figueras et al., 2007b; Janda and Abbott, 2010; Figueras and Beaz-Hidalgo, 2015). However, there are also many publications providing support for a causal relation between Aeromonas and enteric disease (Figueras et al., 2007a,b; Janda and Abbott, 2010; Figueras and Beaz-Hidalgo, 2015). Although 10 of the ca. 30 species that comprise the genus have been isolated in association with gastroenteritis, only 4, i.e., Aeromonas caviae, A. veronii, $A$. dhakensis, and A. hydrophila are the dominating species accounting for ca. $96 \%$ of the recovered isolates from this origin in different studies (Figueras and Beaz-Hidalgo, 2015).

Consumption of contaminated water and food are considered the main routes of transmission and there are few reports of well documented outbreaks that include information about the ingested doses of Aeromonas (Krovacek et al., 1995; Granum et al., 1998; Zhang et al., 2012). An association between diarrheal cases and consumption of untreated or contaminated drinking water has been established in several occasions (Holmberg et al., 1986; Martin Delgado et al., 2001; Khajanchi et al., 2010; Pablos et al., 2011; Ventura et al., 2015). Aeromonas was isolated from drinking water and stools in some patients that were diagnosed with traveler's diarrhea among the tourists that visited specific hotels in the coastal area of Tenerife and Canary Islands in Spain (Martin Delgado et al., 2001). Failures in the drinking water distribution system caused fecal contamination of the water, as revealed by the presence of bacterial indicators of fecal pollution, high concentrations of organic matter and the detection of Aeromonas (Martin Delgado et al., 2001). Once the water distribution system was repaired the incidence of diarrhea among the tourist population returned to normal. Even a recurrent case of Aeromonas bacteremia has been attributed to the consumption of contaminated well water (Katz et al., 2015). Use of contaminated water can cause secondary contamination of food products, and this can be the source of food-borne outbreaks (Altwegg et al., 1991; Krovacek et al., 1995; Granum et al., 1998; Figueras and Borrego, 2010; Wadhwa et al., 2012; Zhang et al., 2012; Figueras and Beaz-Hidalgo, 2014).

It has become generally accepted that only a subset of Aeromonas strains can cause gastroenteritis in humans (von Graevenitz, 2007; Janda and Abbott, 2010; Grim et al., 2014). However, nowadays it is clear that infection is a complex process in which not only the virulence of the colonizing strain is important, but also its interaction with other microbes that are present in the gut, as co-infecting pathogens or in the natural microbial ecosystem, together with the specific physiological status of the host (Lund and O'Brien, 2011; Leggett et al., 2012; Beaz-Hidalgo and Figueras, 2013; Mosser et al., 2015; Ribet and Cossart, 2015; Denny et al., 2016). In fact, the overall crosstalk and interactions between commensal bacteria, enteric pathogens, and host physiology is what is considered crucial to the establishment and progression of intestinal disease (Leggett et al., 2012; Ribet and Cossart, 2015).

Challenge studies have been essential, to establish a causal relation between exposure and health effects and for quantifying the dose response relation (Teunis et al., 1996; Teunis and Havelaar, 2000). However, some outbreak reports include data on the numbers of person exposed, and even information allowing an estimate of the magnitude of the dose involved. Such outbreaks may be treated as 'natural experiments or natural exposure, comparable to a challenge experiment with a single dose group (Teunis et al., 2004, 2008, 2012b). Both data sources have been successfully used in several studies that investigated the potential of human pathogens different from Aeromonas for producing colonization (infectivity) and acute illness (pathogenicity) (Teunis et al., 2005, 2010).

In the present study we use dose response models for microbial infection and acute illness (Teunis et al., 1999; Teunis and Havelaar, 2000) to re-assess the Morgan et al. (1985) challenge study and combine these data with outbreak investigations to determine the dose response relation for producing infection and acute enteric disease by Aeromonas spp. Furthermore, to illustrate the outcomes, we estimate the risk associated with waterborne exposure to these bacteria using reference concentrations found in different water or food matrices. In addition we provide evidence in support of Aeromonas acting as a true enteropathogen.

\section{MATERIALS AND METHODS}

\section{Data Used in the Dose Response Model}

The volunteer protocols for the Morgan et al. (1985) human challenge study were approved by the institutional review boards of the University of Texas Health Science Center, Baylor College of Medicine, The Methodist Hospital and the General Clinical Research Center, and as common in a dose response study, did not include a non-exposed control group. The five different 
Aeromonas strains that were administered orally in a bicarbonate solution to 57 adult healthy human volunteers at a range of doses and the outcomes obtained (Morgan et al., 1985) are summarized in Table 1. A prechallenge stool was cultured for enteropathogens from all volunteers before admission, and volunteers abstained from eating and drinking $90 \mathrm{~min}$ before and after oral challenge. Volunteers were followed up with a daily physical examination to determine symptoms of gastroenteritis (defined as 2 or more unformed stools in $24 \mathrm{~h}$ accompanied by any symptoms of enteric disease). Infection was defined as fecal shedding of the inoculated strain detected by analysis of the volunteer's feces.

One of the subjects who was challenged with strain 3647 developed acute enteric symptoms, while no shedding of the inoculated strain could be detected. This outcome could indicate a false positive for symptoms, if the enteric symptoms in this subjects were caused by something different, unrelated to the challenge. It could also be a false negative infection, where this subject had been colonized without detectable pathogen shedding. Such false negative shedding has been reported in other challenge studies (DuPont et al., 1969), possibly indicating intermittent shedding or low sensitivity of the detection method. Given the setting of inoculation with a high dose, we consider the second alternative (false negative infection) most plausible. In addition to these clinical experiment data, a literature review was done to collect data on acute enteric illness caused by Aeromonas due to natural exposures or outbreaks (here called "natural experiments"). Only studies where sufficient information was reported to allow a dose response assessment were selected. Four "natural experiments" with these characteristics were found: 3 foodborne outbreaks (Krovacek et al., 1995; Granum et al., 1998; Zhang et al., 2012) and an incident where a laboratory

TABLE 1 | Strains of Aeromonas and doses challenged to different groups of volunteers (data from Morgan et al., 1985).

\begin{tabular}{|c|c|c|c|c|}
\hline \multirow[b]{2}{*}{ Strain } & \multirow{2}{*}{$\begin{array}{l}\text { Dose } \\
\text { (cfu) }\end{array}$} & \multicolumn{3}{|c|}{ Volunteers } \\
\hline & & Challenged & Infected & Illness \\
\hline \multirow[t]{5}{*}{$6 Y$} & $2 \times 10^{4}$ & 4 & 1 & 0 \\
\hline & $1 \times 10^{6}$ & 4 & 1 & 0 \\
\hline & $7 \times 10^{7}$ & 4 & 4 & 0 \\
\hline & $3 \times 10^{9}$ & 4 & 3 & 1 \\
\hline & $4 \times 10^{10}$ & 4 & 2 & 0 \\
\hline \multirow[t]{2}{*}{ B158 } & $6 \times 10^{4}$ & 4 & 0 & 0 \\
\hline & $2 \times 10^{7}$ & 4 & 0 & 0 \\
\hline \multirow[t]{4}{*}{3647} & $1 \times 10^{7}$ & 4 & $1^{a}$ & 1 \\
\hline & $4 \times 10^{7}$ & 4 & 0 & 0 \\
\hline & $2 \times 10^{9}$ & 4 & 2 & 0 \\
\hline & $3 \times 10^{10}$ & 4 & 1 & 0 \\
\hline \multirow[t]{2}{*}{ SSU } & $4 \times 10^{8}$ & 4 & 0 & 0 \\
\hline & $5 \times 10^{10}$ & 3 & 0 & 0 \\
\hline \multirow[t]{2}{*}{3284} & $3 \times 10^{8}$ & 3 & 0 & 0 \\
\hline & $1 \times 10^{10}$ & 3 & 0 & 0 \\
\hline
\end{tabular}

according to Morgan et al. (1985), there was not infection but it was only acute illness, however, this could be either a false negative or a false positive (as explained in the materials and methods). Given the setting of inoculation with a high dose, we consider the first alternative (false negative infection) most plausible. worker had accidentally ingested a pure culture of $10^{9}$ Aeromonas (Carnahan et al., 1991). The data used from those studies are summarized in Table 2. To our knowledge these are the only reports that include numbers of exposed subjects and numbers of acute cases, and information on exposure, either as estimated numbers of ingested bacteria or as amount of the contaminated food product consumed (Table 2). The foodborne outbreak reported by Zhang et al. (2012) showed a dose response relation between the amounts of food consumed (cucumber salad) and the observed attack rate.

\section{Dose Response Assessment}

Conceptually, three stages may be identified when a subject is challenged: exposure, infection, and (acute) illness. For exposure, it is important to realize that many microbial pathogens are highly infectious. Whenever a person ingests a quantity of contaminated food or drink, they only need to swallow few pathogenic particles to become infected. If the concentration of pathogens in the contaminated food or drink is low, the probability that the ingested portion did not contain any pathogens may be substantial. If (and only if) a person is exposed, there is a non-zero probability that any of the ingested pathogens survives all host barriers and succeeds in colonizing host tissues (i.e., infection is conditional on exposure). Likewise, if (and only if) a person is infected, there is a non-zero probability that the colonizing pathogen expresses pathogenicity producing damage of host tissues leading to symptoms of (acute) illness (i.e., illness is conditional on infection). Thus, there are two conditional probabilities: the probability of infection given exposure, and the probability of (acute) illness given infection. Both probabilities may be dose dependent, so that there are two dose response relations: one for infection, and the second for illness among infected subjects. Mathematical details for these two dose response relations are given in the Supplementary Material, where it is also indicated that all analyses were performed using JAGS (v4.2.0), with post-processing (graphs and additional statistics) in $\mathrm{R}$ (v3.3.1).

The parameters for these dose response relations, characterizing susceptibility to infection and to illness when infected, were estimated in a hierarchical framework. Each strain in the challenge study was treated as a separate trial, leading to strain-specific dose response relations for infection and illness. Similarly, each "natural experiment" was treated separately, as described by its own distinct dose response relations, for infection and illness. In the hierarchical framework, the variation in the parameters among these separate dose response relations is described by a (joint) distribution, representing the "group" pattern for all studies combined (Teunis et al., 2008). Hence, using all studies combined, it is possible to make predictions of the dose response relations for infection and illness, for any Aeromonas as representing a random sample from a population characterized by the combined studies shown in Tables $\mathbf{1}$ and $\mathbf{2}$.

In a subsequent analysis the challenge studies and the natural experiments were compared by assigning each of these two categories different susceptibilities to infection and illness. These separate predictions of the dose response relations for the 
TABLE 2 | Dose and/or concentrations of Aeromonas found in the ingested matrices and number of exposed and cases showing acute gastrointestinal symptoms in outbreaks or natural experiments.

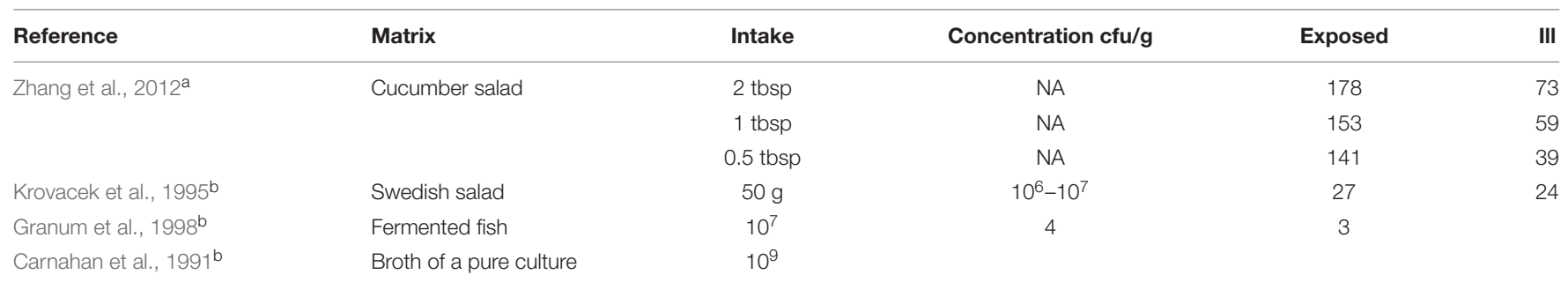

$N A=$ not available; tbsp = table spoon; a Intake of food (cucumber salad) with unknown concentration, so the concentration was estimated in this study (Figure 1). ${ }^{\mathrm{b}}$ The concentration is the dose given by the authors.

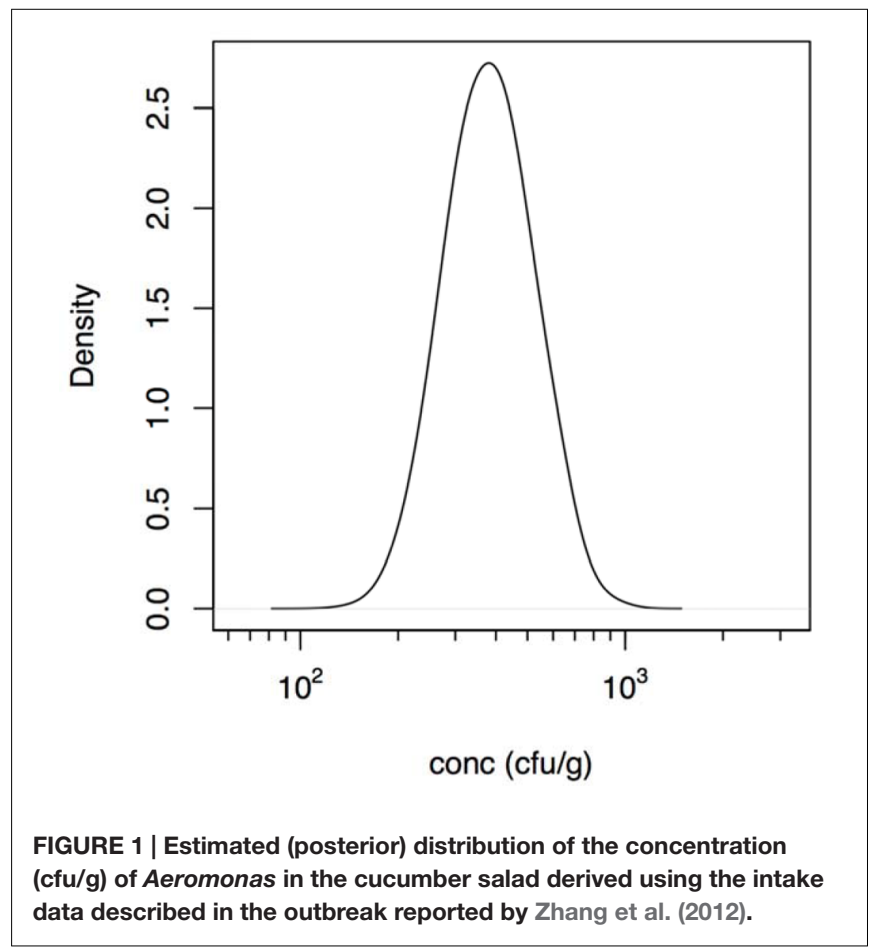

challenge study strains and for the natural exposure events may thus be compared.

Note that in the foodborne outbreak described by Zhang et al. (2012) the intake of contaminated food was reported, but not the concentration of bacteria or its possible inhomogeneous distribution in that food due to clustering occurrence. Assuming the dose is proportional to the amount consumed, the concentration of bacteria may be added as another parameter (Teunis et al., 2005). This allows estimation of the mean dose (show in Figure 1) even in case there is heterogeneity because of an uneven distribution of bacteria (clustering) in the contaminated food. Unfortunately, the information available does not allow quantitative characterization of this heterogeneity as done in previous studies (Teunis et al., 2008).

\section{Estimation of Risk}

To illustrate the use of the inferred dose response models, Monte Carlo samples of the dose response parameters for infection and (conditional) illness were used to calculate risks of infection and (acute enteric) illness for a few exposure scenarios. The probabilities of infection and illness resulting from exposure to low, medium, and high doses $\left(10,1000\right.$, and $\left.10^{6} \mathrm{cfu}\right)$ of Aeromonas were calculated, using the dose response models for infection and illness (see annex Supplementary Material). The selected scenarios represent concentrations of Aeromonas commonly found in drinking water distribution systems or food products like milk, meat products or shellfish (Abeyta et al., 1986; Austin et al., 1996; Borrell et al., 1998; Figueras et al., 2005; Egorov et al., 2011; Wadhwa et al., 2012; Robertson et al., 2014) or contaminated water (rivers, lakes) with treated or untreated wastewater (Austin et al., 1996; Borrell et al., 1998; McMahon and Wilson, 2001; Janda and Abbott, 2010; Latif-Eugenín, 2015; Fernandez-Cassi et al., 2016).

\section{RESULTS}

\section{Dose Response Assessment}

The calculated estimated concentration of Aeromonas in the cucumber salad that was the vehicle for exposure in the outbreak reported by Zhang et al. (2012), is shown in Figure 1 and ranged between 200 and $1000 \mathrm{cfu} / \mathrm{g}$. So the likely dose in that outbreak was lower than the doses in the challenge study (see Supplementary Material for details).

In an initial analysis the data from the challenge study and the outbreaks (natural experiments or natural exposure events) were assumed to be similar and were represented by single joint distributions (Figure 2) that showed the susceptibility to infection (i.e., the probability of infection per ingested bacteria) and to illness (i.e., the probability of illness per ingested bacteria). This unsegmented approach resulted in a single predicted doseresponse relation for illness, as show in Figure 2, with a broad posterior range in illness probabilities, indicating substantial heterogeneity in illness risk. In Figure 2 it can be observed that the data from the "natural experiments" (i.e., outbreaks or natural exposure events) cluster at higher illness probabilities compared to the challenge study data. Based on these different outcomes, a refined model was set up, with separate categories for the challenge study and the "natural experiments" that represented two different distributions of susceptibility. The resulting separate dose response models for the challenge study 


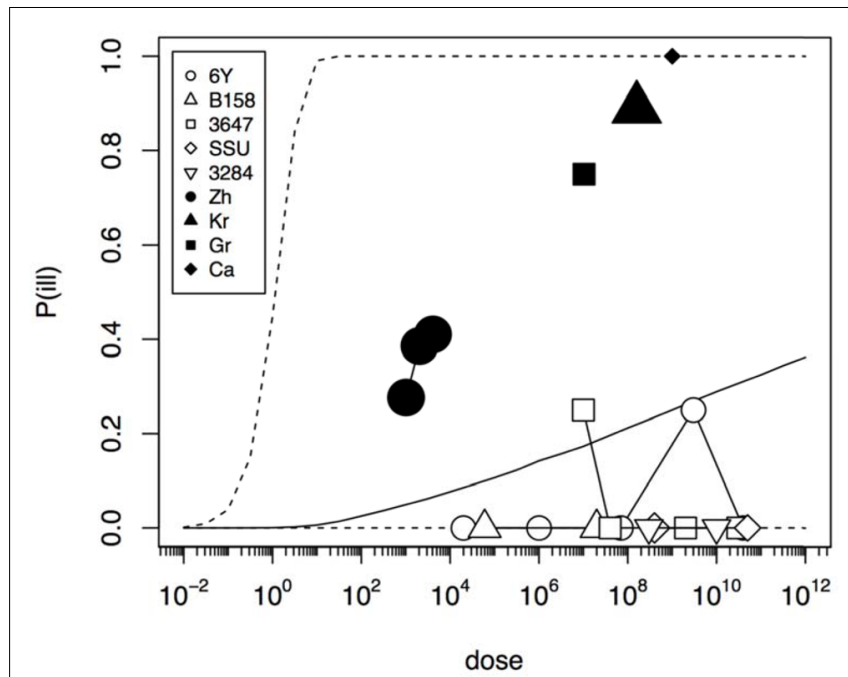

FIGURE 2 | Predicted dose response relation for acute enteric illness assuming a single class of infectivity and pathogenicity. Median probability of illness (solid black line) and 95\% predictive intervals (dotted lines). The five strains (6Y, B158, 3647, SSU, and 3284) used in the challenge study show a low virulence (data in Table 1), and the four natural experiments ( $\mathrm{h}$ h = Zhang et al., $\mathrm{Kr}=$ Krovacek et al., $\mathrm{Gr}=$ Granum et al., $\mathrm{Ca}=$ Carnahan et al.) show a high virulence (data in Table 2). Plot symbols show observed fractions, data from the same strain/experiment are connected. Sizes of symbols indicate numbers of subjects exposed.

and "natural exposure events" were markedly different, as shown in Figure 3. For the challenge study, ingestion of even very high doses of $10^{12} \mathrm{cfu}$ results in low risk of acute illness (approximately 0.1, 95\% range 0-0.45) as shown in Figure 3. Note, however, that even this low risk is caused by only two positive responses, with two different strains (3647 and 6Y), out of a total of 57 subjects exposed. In an alternative analysis where the symptomatic case for strain 3647 was considered a false positive, the resulting dose response models (not shown here) were virtually identical to those in Figures 2 and 3. Of those exposed, 15 were infected, indicating that infection was a lot more common than symptomatic acute illness, and also that pathogenicity may have been different among strains. For the "natural exposure events" the illness risk was much higher (approximately 0.5, 95\% range 0.05-1.0), even at a comparatively low dose of $10^{3} \mathrm{cfu}$ (Figure 3, lower graphic).

Figure 4 shows infection dose response relations for the challenge study and the "natural exposure" data. As infection is a condition for illness, the probability of infection must be at least as high as the illness probability (compare Figures 3 and 4). The natural exposure event data do not include any observation of infections, as this is a covert outcome (it cannot be observed directly), but because illness depends on infection the probability of infection can still be estimated. As the illness risk was high in these "natural experiments", the infection probabilities must be high as well, as shown in Figure 4.

The estimated susceptibilities for infection and illness were illustrated also by the doses required to cause a $1 \%$ probability of infection for each of the challenge strains, and separately for each
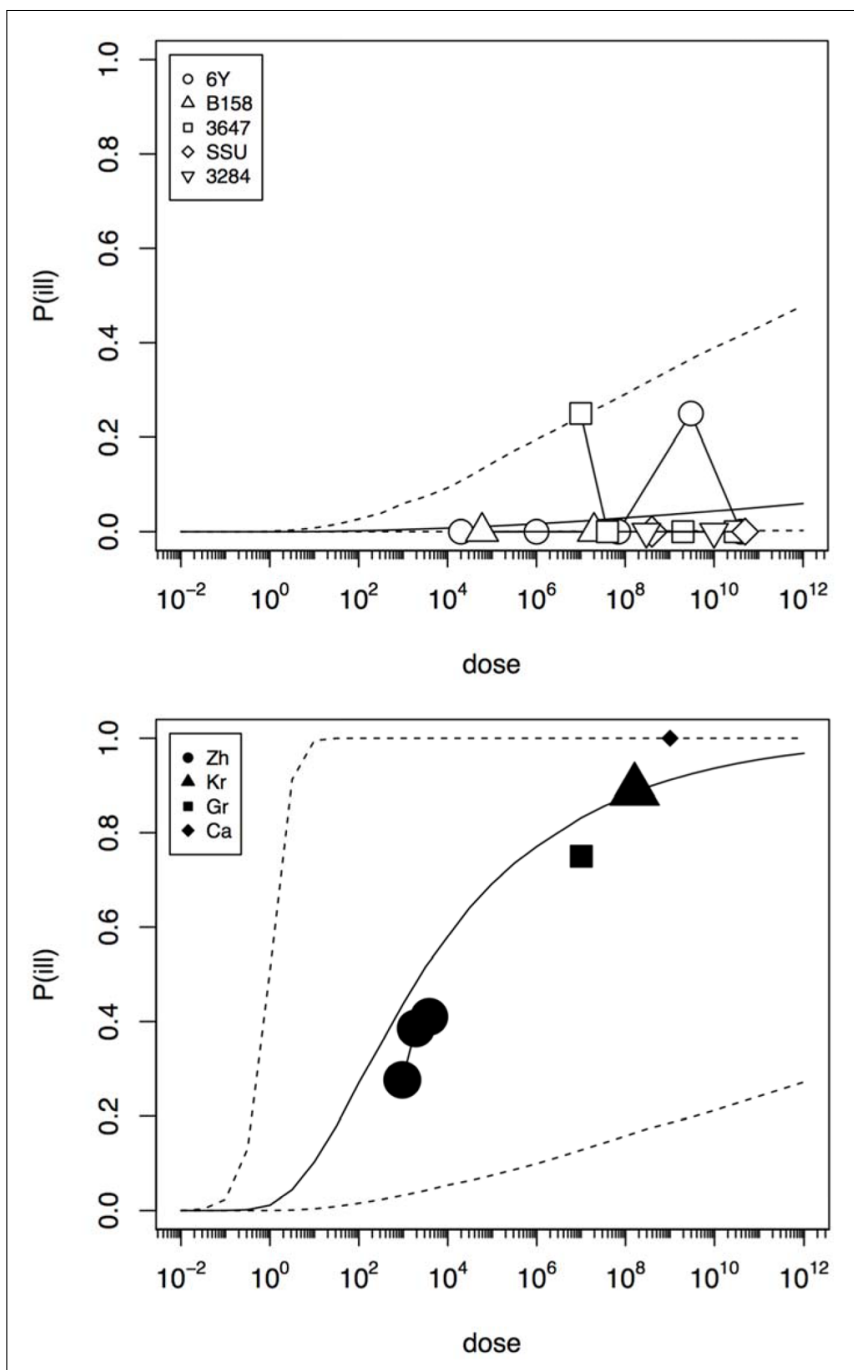

FIGURE 3 | Predicted dose response relations for acute enteric illness, derived from the five strain (6Y, B158, 3647, SSU, and 3284) of the challenge study (upper graphic showing a low susceptibility) and the four natural experiments $(\mathrm{Zh}=\mathrm{Zhang}$ et al., $\mathrm{Kr}=\mathrm{Krovacek}$ et al., $\mathrm{Gr}=$ Granum et al., $\mathbf{C a}=$ Carnahan et al., lower graphic showing a high susceptibility). Median probability of illness (solid black line) and 95\% predictive intervals (dotted lines). Plot symbols show observed fractions, data from the same strain/experiment are connected. Sizes of symbols indicate numbers of subjects exposed.

of the "natural exposure events" (Table 3; Figure 5). Estimates for the two prediction categories: low susceptibility for the challenge studies and high susceptibility for the "natural experiments" are shown in Figure 5 for infection and for illness risks. An outcome of $1 \%$ risk was chosen here because in the challenge studies the risk was so low that calculation of 50\% infectious (and illness) doses results in unrealistically high numbers. Mean and median, as well as a 95\% range are also given in Table 3.

\section{Estimation of Risk}

To illustrate the application of the dose response models derived here, a small risk study was set up, using a simple scenario 


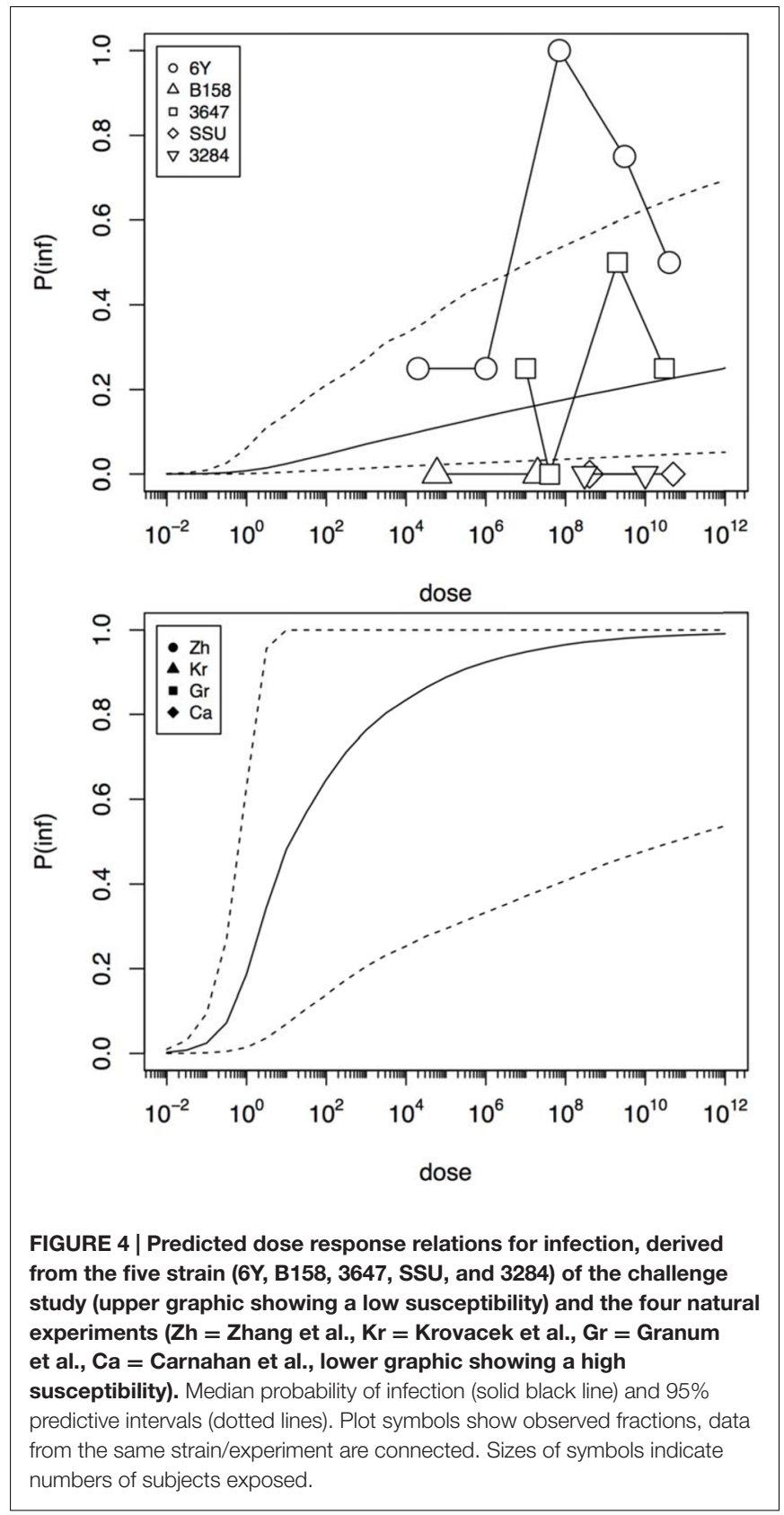

of three different, fixed doses, representing low, medium, and high exposure. Figure 6 shows the resulting risks of Aeromonas infection and illness. It is clear that using the challenge study dose response relation, the illness risk remains small, even for exposure to considerable doses of Aeromonas. On the other hand, the natural exposure events dose response relation produces high illness risks, even when the dose is moderate or low.

\section{DISCUSSION}

The major clinical manifestation produced by Aeromonas is diarrhea affecting principally young children and immunocompromised patients (Janda and Abbott, 2010; Figueras and Beaz-Hidalgo, 2015). The single available human challenge study for Aeromonas shows low susceptibility, in particular to developing acute enteric symptoms with only $2 / 57$ (3.5\%) challenged being positive (Table 1; Morgan et al., 1985). In contrast, the "natural exposure events" indicate that a high risk of illness may be associated with exposure to Aeromonas even at moderate to low doses. Thus, host susceptibility may be a strong determinant for the illness risk of Aeromonas sp. The dose response assessment presented in this study corroborates this conclusion (median 1\% illness dose was $\sim 1.4 \times 10^{4}$ times higher in "the natural exposure events" compared to the clinical challenge), and shows how these two subsets of illness data lead to different estimates of susceptibility.

Although invaluable for understanding infection and pathogenesis, clinical challenge may not quantitatively represent natural infection that occur during an outbreak. Clinical challenge may underestimate the risk because pathogen inocula may decrease in virulence because of safety testing, often requiring repeated culture in the laboratory. Likewise, volunteers are selected for being healthy and immunocompetent to reduce the risk of serious complications. Conversely, outbreaks select for virulent pathogen strains and susceptible hosts, as this increases the probability that a cluster of cases is detected (Teunis et al., 2004). Consequently, challenge studies and outbreaks may both be biased, in opposite directions: each may represent an extreme in the continuum of dose response relations as discussed earlier (Teunis et al., 2005, 2010; Thebault et al., 2013). Outbreaks or natural exposure events may select for highest susceptibility in the affected host population producing a high illness risks, even when the dose is moderate or low. For instance in the food outbreak that produced acute diarrhea in over 200 college students' described by Zhang et al. (2012) the estimated concentration of Aeromonas in the cucumber salad determined in the present study ranged from 200 to $1000 \mathrm{cfu} / \mathrm{g}$ (Figure 1). This estimated concentration assumed a homogeneous distribution of bacteria (Teunis et al., 2005) in the cucumber salad. As the origin of the contamination in this outbreak was attributed to rinse water of the vegetables, contaminated with sewage, occurrence of bacteria may not have been severely clustered. In case there is severe clustering, some subjects would have been exposed to high doses, while others would not have been exposed at all. Only when there is extreme clustering, the slope of the dose response relation is affected, not its location and the mean dose remains proportional to the amount of contaminated food that was ingested (Teunis et al., 2008, 2012a). As no information regarding clustering in the contaminated cucumber salad was provided by Zhang et al. (2012), any assumptions regarding clustering would be speculation.

Little is known about who is susceptible to any specific strain or pathogen in the population at large, or how many of the already considered susceptible like infants or immunocompromised would develop acute illness. It has been estimated that in developed countries the part of the population that could be susceptible to acquiring a foodborne disease would be near 20\% (Lund and O'Brien, 2011; Lund, 2015). Challenge 


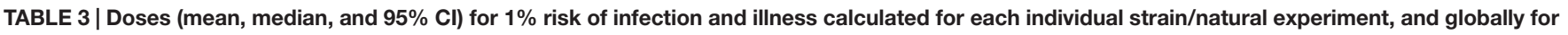
all strain/natural experiments (corresponding to the low/high susceptibility situations).

\begin{tabular}{|c|c|c|c|c|c|c|}
\hline & \multicolumn{3}{|c|}{$1 \%$ Infectious dose } & \multicolumn{3}{|c|}{$1 \%$ Illness dose } \\
\hline & Mean & Median & $95 \% \mathrm{Cl}$ & Mean & Median & $95 \% \mathrm{Cl}$ \\
\hline $6 Y^{a}$ & 1.04 & 0.56 & $0.07-4.95$ & $1.47 \times 10^{4}$ & 60.8 & $4.19-5.09 \times 10^{3}$ \\
\hline $\mathrm{B} 158^{\mathrm{a}}$ & 1893.6 & 2.58 & $0.15-126.5$ & $1.90 \times 10^{9}$ & $4.97 \times 10^{5}$ & $126.0-2.67 \times 10^{10}$ \\
\hline $3647^{a}$ & 2.69 & 1.27 & $0.12-14.6$ & $2.94 \times 10^{8}$ & 8400.7 & $53.6-1.54 \times 10^{9}$ \\
\hline sSua & 412.1 & 3.20 & $0.14-191.4$ & $2.48 \times 10^{9}$ & $1.22 \times 10^{6}$ & $215.1-3.77 \times 10^{10}$ \\
\hline $3284^{a}$ & 2839.6 & 2.93 & $0.14-163.5$ & $2.26 \times 10^{9}$ & $6.10 \times 10^{5}$ & $189.6-3.30 \times 10^{10}$ \\
\hline $\mathrm{Zh}^{\mathrm{b}}$ & 0.14 & 0.05 & $0.01-0.68$ & 3.94 & 1.28 & $0.054-22.67$ \\
\hline $\mathrm{Kr}^{\mathrm{b}}$ & 0.10 & 0.04 & $0.01-0.55$ & 1.98 & 0.82 & $0.063-10.88$ \\
\hline $\mathrm{Gr}^{\mathrm{b}}$ & 0.12 & 0.04 & $0.01-0.70$ & 3.90 & 0.94 & $0.058-24.95$ \\
\hline $\mathrm{Ca}^{\mathrm{b}}$ & 0.12 & 0.04 & $0.01-0.68$ & 331.8 & 0.85 & $0.064-31.76$ \\
\hline Low Susc ${ }^{c}$ & 385.1 & 1.67 & $0.10-61.2$ & $7.61 \times 10^{8}$ & $1.24 \times 10^{4}$ & $11.76-7.60 \times 10^{9}$ \\
\hline High Susc ${ }^{C}$ & 0.12 & 0.04 & $0.01-0.73$ & 21.88 & 0.90 & $0.064-50.67$ \\
\hline
\end{tabular}

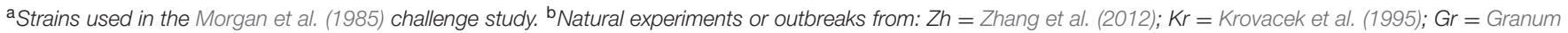

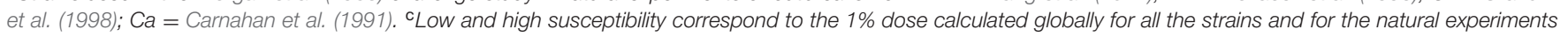
or outbreaks, respectively.

studies, on the other hand, tend to select for low susceptibility, and may represent the opposite extreme of the susceptibility spectrum. The results presented here for Aeromonas appear to support the same conclusion, where the outbreaks represent a worst case situation, while most challenged exposures result in low risk of infection and illness.

As indicated before the results of the Morgan et al. (1985) challenge study have been used to indicate that Aeromonas is not a true gastrointestinal pathogen (von Graevenitz, 2007; Janda and Abbott, 2010). However, it must be noted that 15 out of 57 (26\%) were infected. The occurrence of few illness outcomes is not rare in human challenge studies. For instance in a challenge study for Giardia lamblia 40 subjects were challenged, with doses up to $10^{6}$ cysts resulting in 21 infected subjects, yet not a single of these infected subjects showed any symptoms of enteric illness (Rendtorff, 1954). Nevertheless, the significance of Giardia as a human enteric pathogen is not doubted. The same may be found for Plesiomonas shigelloides with 22 subjects challenged, resulting in eight infected and none of these becoming ill (Herrington et al., 1987). The 3.5\% acute diarrhea incidence of the Morgan et al. (1985) study is similar to the $2 \%$ of adult diarrhea incidence found in several studies (Svenungsson et al., 2000; Vila et al., 2003; Figueras, 2005; Senderovich et al., 2012; Figueras and Beaz-Hidalgo, 2015).

The above analysis suggests that the natural exposures involve much lower doses than the challenge study. Notwithstanding the low risk predicted from the challenge study data, the same study suggests that the risk of infection is not extremely low, in particular for two of the strains 6Y and 3647 (Morgan et al., 1985). This means that the risk cannot be ignored, even though few cases of acute diarrhea were observed. Janda and Abbott (2010) underlined that the strain SSU (CDC diarrheal isolate), which is probably the most well-characterized Aeromonas strain with respect to virulence factors (i.e., carriage and expression of enterotoxin genes, and potential colonization factors see studies directed by Dr. Chopra, i.e., Grim et al., 2014 and
Ponnusamy et al., 2016, and references therein) did not produce infection (colonization) nor diarrhea in the challenge study, so this cannot support the idea that the strains were wrongly selected. However, it is still possible that a critical virulence or colonization factor could have been lost when subculturing the original strains (Morgan et al., 1985; Janda and Abbott, 2010). Experimental studies in a murine animal model showed that the injection of strain SSU generated the death of all mice in 48 h (Grim et al., 2014; Ponnusamy et al., 2016). Strain SSU has been recently identified as Aeromonas dhakensis, a highly virulent species (Beaz-Hidalgo et al., 2013; Morinaga et al., 2013; Figueras and Beaz-Hidalgo, 2015), instead of A. hydrophila as originally thought (Morgan et al., 1985). It is now known that the importance attributed to A. hydrophila is due to misidentification of the majority of strains as belonging to this species, using phenotypic identification systems (Soler et al., 2003; Figueras, 2005; Beaz-Hidalgo et al., 2010; Morinaga et al., 2013). In fact even $30 \%$ of the genomes deposited at the GenBank database with the name of $A$. hydrophila do not belong to this species (Figueras et al., 2014; Beaz-Hidalgo et al., 2015). Old literature on clinical aeromonads limited the identification only to three species, i.e., A. hydrophila, A. sobria (the correct terminology for the clinical strains is $A$. veronii biovar sobria) and A. caviae (Janda and Abbott, 1998; Figueras, 2005; von Graevenitz, 2007). However, strains under these names may belong to other species (Janda and Abbott, 1998; Soler et al., 2003; Figueras, 2005). For instance in the Morgan et al. (1985) study among the five strains named $A$. hydrophila three (6Y, B158, and 3284) were suspected to belong to A. sobria (A. veronii biovar sobria), and strain SSU, as commented above, corresponds to A. dhakensis. A recent review showed that when using molecular identification methods ca. $96 \%$ of the recovered isolates (313/327) from human feces in different studies belong to 4 species: A. caviae (37.6\%), A. veronii (27.2\%), A. dhakensis (16.5\%), and A. hydrophila (14.5\%) (Figueras and Beaz-Hidalgo, 2015). A higher prevalence of $A$. caviae in diarrhea cases over the other species is reported 

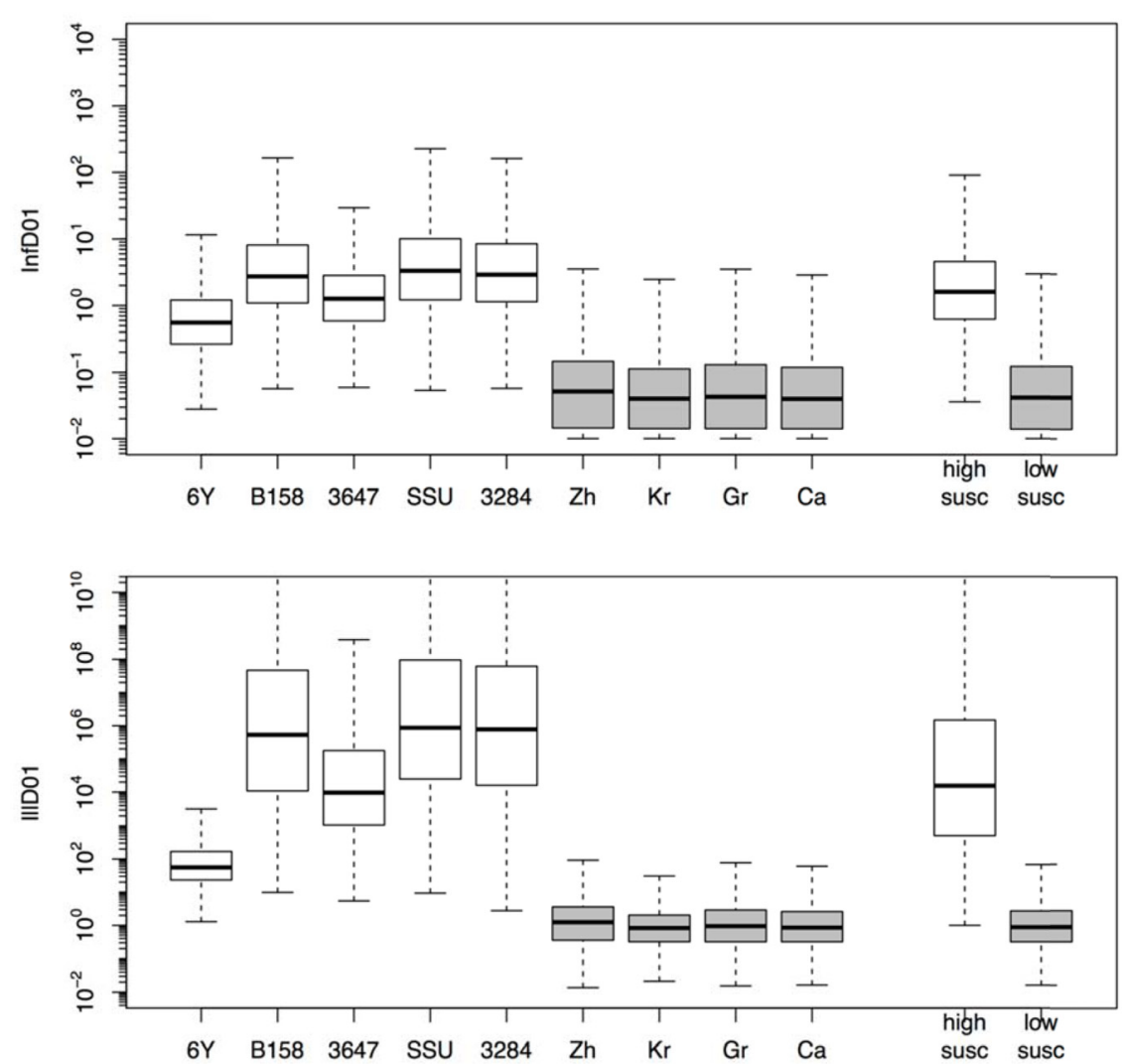

FIGURE 5 | Box plots showing the estimated doses required for 1\% probability of infection (InfD01, upper graphic) and illness (IIID01, lower graphic). Results in both graphics are provided for each individual strain (6Y, B158, 3647, SSU, and 3284) and experiment (Zh = Zhang et al., Kr = Krovacek et al., $\mathrm{Gr}=$ Granum et al., $\mathrm{Ca}=$ Carnahan et al.) and globally for all strains (i.e., low susceptibility) and natural experiments (i.e., high susceptibility).

also in previous reviews (von Graevenitz, 2007). However, studies on traveler's diarrhea where an adult population is involved are dominated by A. veronii (Vila et al., 2003; von Graevenitz, 2007 and references therein). Also in a recent study performed by Chen et al. (2015) in and adult population from Taiwan A. veronii was the prevailing species (54.6\%) followed by A. caviae $(27.6 \%)$, and with one strain of each of the species A. dhakensis $(9.1 \%)$ and A. sanarellii (9.1\%). Interestingly one patient in the latter study had a history of eating lettuce with salad prior to the illness. In a recent study tomatoes and parsley irrigated with the same water showed the same genotype of a strain of $A$. sanarellii and the same occurred for an $A$. caviae strain recovered from the water and the irrigated lettuces (Latif-Eugenín, 2015).

As we commented in the introduction it is generally assumed that the virulence of Aeromonas is multifactorial and that only a subset of Aeromonas strains is capable of causing gastroenteritis in humans (von Graevenitz, 2007; Janda and Abbott, 2010; Beaz-Hidalgo and Figueras, 2013; Figueras and Beaz-Hidalgo, 2014; Grim et al., 2014; Ponnusamy et al., 2016). In fact some Aeromonas strains possess several virulence toxins and secretion systems, among which the Type III secretion system and the Shiga toxin genes are similar to those present in other important pathogenic bacteria (Figueras et al., 2007a; Alperi and Figueras, 2010; Figueras and Beaz-Hidalgo, 2014). The large variation observed among Aeromonas strains maybe linked to the site where the virulence is expressed (Leggett et al., 2012). For instance carriage of virulence genes that have a local action within the host cells like, i.e., the injection of toxins through the Type III or Type IV secretion systems may generate a more virulent response than other strains that exert virulence at more distant sites (Leggett et al., 2012). The latter occurs when pathogens cause secretion of proteins binding to host cells (i.e., immune modulators delivered by the general secretary pathway, or by Type I, II, and V secretory systems). This different pathway or the synergetic effect of such pathways may lead to differences in infective doses (Leggett et al., 2012). In addition the host susceptibility is very relevant, now we know that the genetic polymorphism in the host population determines the variability observed in the type or intensity of responses against the encountered specific pathogens ranging from asymptomatic infections to fatal disease (Ribet and Cossart, 2015).

The concentrations of Aeromonas that were selected in the exposure scenarios to assess risk of infection, were those that can normally be found in oysters, i.e., MPN 9.3/100 g or $10 \mathrm{cfu} / 100 \mathrm{ml}$ have been reported in several drinking water studies (Abeyta et al., 1986; Austin et al., 1996; Figueras et al., 2005; Egorov et al., 2011; Robertson et al., 2014) or for instance in Milk $\left(1-2 \times 10^{3}\right)$ or meat products $\left(10^{2}-10^{3}\right)$ as found by Borrell et al. (1998). 

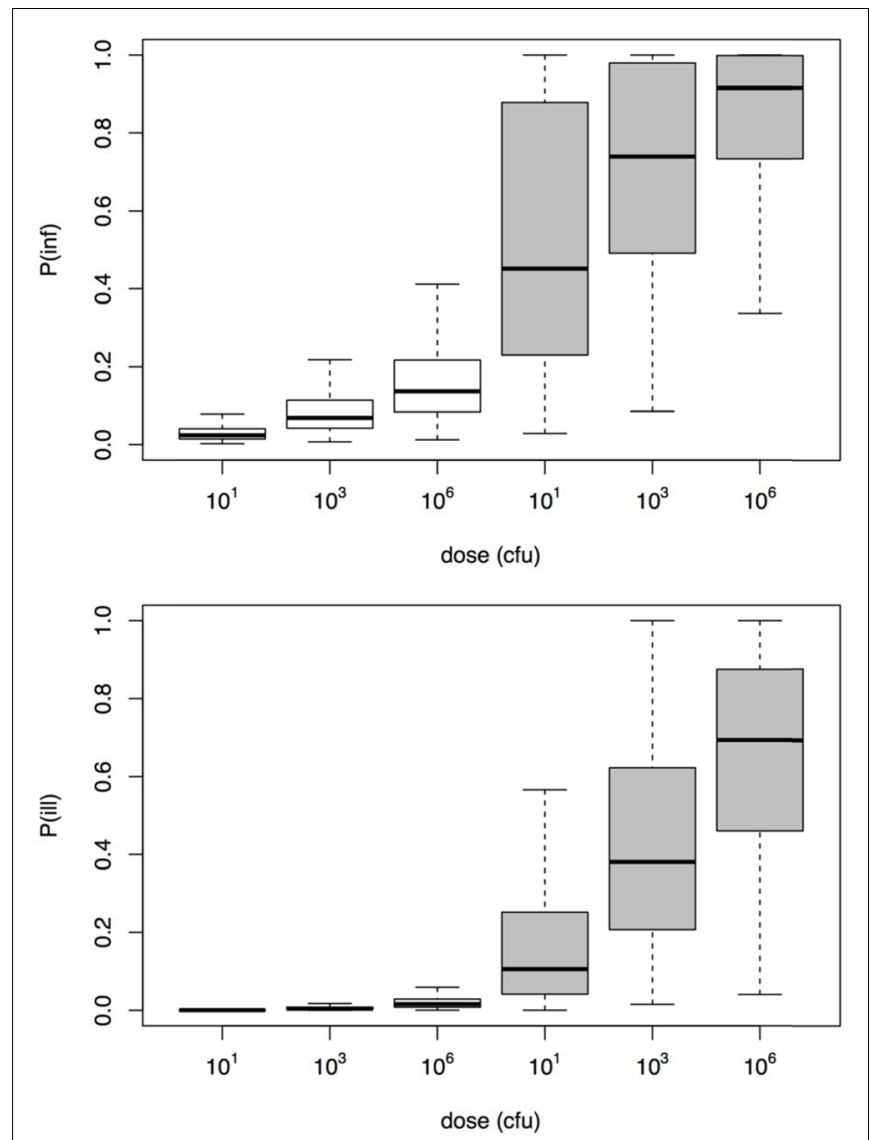

FIGURE 6 | Risk of infection (upper graphic) and illness (lower graphic) for the three different dose scenarios Aeromonas exposure (10, 1000, and $10^{6} \mathrm{cfu}$ ).

In fact a MPN 9.3/100g was the concentration of Aeromonas found in oysters considered to be the source of an outbreak that affected 472 persons suffering from gastroenteritis in Louisiana (Abeyta et al., 1986). Concentrations as high as $10^{5}$ or $10^{6}$ $\mathrm{cfu} / 100 \mathrm{ml}$ can be found in treated wastewater and in reclaimed waters used for irrigation of vegetables (Austin et al., 1996; McMahon and Wilson, 2001; Janda and Abbott, 2010; FernandezCassi et al., 2016). Such sources could contaminate ready to eat vegetables and pose a risk for human health as was recently demonstrated finding the same genotype of Aeromonas in the irrigated water and in the irrigated vegetables as commented above (Latif-Eugenín, 2015).

The dose response assessment reported here shows that when Aeromonas is present, the probability of infection may not be negligible, and in a susceptible host, there may be a high risk of acute enteric illness. It is therefore important to determine how rare it is for a host to be highly susceptible. Outbreaks or other natural experiments are hard to find. This may be because they are so rare, but there could be underreporting because Aeromonas is often not considered among the microbes to be analyzed during investigation of outbreaks of infectious gastroenteritis (Janda and Abbott, 2010; Figueras and Beaz-Hidalgo, 2015). In fact the recognition of Aeromonas in the clinical setting occurs accidentally on routine enteric isolation media designed for other enteropathogens like XLD (MacConkey, Xylose Lysine Dextrose Agar), SS (Salmonella-Shigella Agar), or CIN (CefsulodinIrgasa-Novobiocin Agar) as described earlier (Figueras, 2005; Janda and Abbott, 1998, 2010; Figueras and Beaz-Hidalgo, 2015).

The carriage of an infectious microorganism in the general population cannot be easily extrapolated from (observable) symptomatic cases, because of underreporting, but also because in the population at large, a great majority of the infections may remain asymptomatic. Biomarker studies using serum antibodies for estimating infection (seroconversion) rates in the general population have shown that for Campylobacter there may be more than 1,000 asymptomatic infections for every notified case of campylobacteriosis (Teunis et al., 2012b, 2013). Similar ratios have been found for Salmonella (Simonsen et al., 2011). Serology could be helpful in determining whether there could be substantial carriage of Aeromonas in the general population. Serological evidence of infection has been provided in some cases, supporting the true enteropathogenicity of Aeromonas (see review by Janda and Abbott, 1998; Crivelli et al., 2001). In fact a specific secretory immunoglobulin A (sIgA) response at the intestinal mucosa against the extracellular products that appeared in the feces of patients with Aeromonas diarrhea was demonstrated by Crivelli et al. (2001).

Prevalence or incidence of HIV, hepatitis C have been estimate using the "evidence synthesis", that is a well-accepted methodology for integrating various sources of data to estimate a quantity of interest for which there are no or limited direct data (McDonald et al., 2015 and references therein). This approach was applied recently to determine the incidence of symptomatic pertussis infection in the Netherlands (McDonald et al., 2015) and may help in determining whether the low numbers of symptomatic cases are consistent with frequent isolation of Aeromonas.

The predicted risks of infection and illness show considerable uncertainty. This is caused by the variation observed between outcomes produced by different strains, but it also results from the small sizes of the exposed groups, in particular in the challenge study (Table 1). Unfortunately it is not likely that there will be more or better data available, to improve the precision of the estimates. Despite the small sample sizes and uncertain doses, what is relevant is that the low and high susceptibility estimates are clearly different.

\section{Aeromonas is a True Enteropathogen}

Arguments used against considering Aeromonas an enteropathogen, i.e., the lack of outbreaks, the non-fulfillment of the Koch's postulates, the low numbers of acute illness in the human challenge study and the lack of animal model have all been addressed with evidence to the contrary in other studies (Chu et al., 2006; Figueras et al., 2007b; von Graevenitz, 2007; Janda and Abbott, 2010; Figueras and Beaz-Hidalgo, 2015). A murine model of Aeromonas diarrhea has been developed (Abuelsaad et al., 2013). The Koch's postulates have been fulfilled by considering the incidental ingestion of $A$. trota by a 
laboratory worker (Carnahan et al., 1991), the challenge study of Morgan et al. (1985) and the outbreak reports included in this study (Krovacek et al., 1995; Granum et al., 1998; Zhang et al., 2012). In addition, even though few outbreaks exist, an epidemiological link has been found between the source of infection and the clinical isolates. The same Aeromonas strains (verified by genotyping) that caused diarrhea were isolated from drinking water (Khajanchi et al., 2010; Pablos et al., 2011), from the consumed shrimp cocktail (Altwegg et al., 1991) and from the household environment (Demarta et al., 2000). Also the same genotype of Aeromonas has been isolated from an HIV/AIDS patient suffering from gastroenteritis and from their household drinking-water (Ramalivhana et al., 2010).

\section{CONCLUDING REMARKS AND PERSPECTIVES}

Combined evidence collated from clinical studies in humans and outbreaks shows that Aeromonas should be treated as a human enteropathogen. Exposure to low doses of Aeromonas sp. may lead to infection, but most infections may remain asymptomatic. Given the omnipresence of Aeromonas in the environment, seroprevalence studies in the general population are needed to reveal frequent carriage. Further investigations are needed to determine the specific combination of host, environment and pathogen factors that lead to the occurrence of acute enteric symptoms (illness) associated with Aeromonas infections. The risk of illness may be considerable,

\section{REFERENCES}

Abeyta, C., Kaysner, C. A., Wekell, M. M., Sullivan, J. J., and Stelma, G. N. (1986). Recovery of Aeromonas hydrophila from oysters implicated in an outbreak of foodborne illness. J. Food Protect. 49, 643-650.

Abuelsaad, A. S., Mohamed, I., Allam, G., and Al-Solumani, A. A. (2013). Antimicrobial and immunomodulating activities of hesperidin and ellagic acid against diarrheic Aeromonas hydrophila in a murine model. Life Sci. 93, 714722. doi: 10.1016/j.lfs.2013.09.019

Alperi, A., and Figueras, M. J. (2010). Human isolates of Aeromonas possess Shiga toxin genes (stx1 and stx2) highly similar to the most virulent gene variants of Escherichia coli. Clin. Microbiol. Infect. 16, 1563-1567. doi: 10.1111/j.14690691.2010.03203.x

Altwegg, M., Martinetti-Lucchini, G., Lüthy-Hottenstein, J., and Rohrbach, M. (1991). Aeromonas-associated gastroenteritis after consumption of contaminated shrimp. Eur. J. Clin. Microbiol. Infect. Dis. 10, 44-45. doi: 10.1007/BF01967100

Austin, B., Altwegg, M., Gosling, P. J., and Joseph, S. W. (1996). The Genus Aeromonas. Chichester: John Wiley \& Sons, Ltd.

Beaz-Hidalgo, R., Alperi, A., Buján, N., Romalde, J. L., and Figueras, M. J. (2010). Comparison of phenotypical and genetic identification of Aeromonas strains isolated from diseased fish. Syst. Appl. Microbiol. 33, 149-153. doi: 10.1016/j.syapm.2010.02.002

Beaz-Hidalgo, R., and Figueras, M. J. (2013). Aeromonas spp. whole genomes and virulence factors implicated in fish disease. J. Fish Dis. 36, 371-388. doi: $10.1111 /$ jfd.12025

Beaz-Hidalgo, R., Hossain, M. J., Liles, M. R., and Figueras, M. J. (2015). Strategies to avoid wrongly labelled genomes using as example the detected wrong taxonomic affiliation for Aeromonas genomes in the GenBank database. PLoS ONE 10:e0115813. doi: 10.1371/journal.pone. 0115813 even when exposed to moderate doses as shown for the studied outbreaks. Therefore, as suggested earlier, patients with underlying malignancies or immunosuppressing conditions should be closely supervised, considering the inherently high risk of invasion and dissemination associated with this population group.

\section{AUTHOR CONTRIBUTIONS}

PT and MF contributed equally to the conception and design of the study, PT performed the mathematical modeling and PT and MF jointly interpreted the data and wrote the manuscript. Both authors approved the final version of the paper.

\section{FUNDING}

This study was party supported by the projects: Aquavalens from the European Union Seventh Framework Program (FP7/20072013) under Grant agreement No.: 311846 , and by projects from Spanish Ministry of Science and Innovation: AGL2011-30461C02-02 and JPIW2013-095-CO3.

\section{SUPPLEMENTARY MATERIAL}

The Supplementary Material for this article can be found online at: http://journal.frontiersin.org/article/10.3389/fmicb. 2016.01395

Beaz-Hidalgo, R., Martinez-Murcia, A. J., and Figueras, M. J. (2013). Reclassification of Aeromonas hydrophila subsp. dhakensis Huys et al. 2002 and Aeromonas aquariorum Martinez-Murcia et al. 2008 as Aeromonas dhakensis sp. nov. comb nov. and emendation of the species Aeromonas hydrophila. Syst. Appl. Microbiol. 36, 171-176. doi: 10.1016/j.syapm.2012.12.007

Borrell, N., Figueras, M. J., and Guarro, J. (1998). Phenotypic identification of Aeromonas genomospecies from clinical and environmental sources. Can. J. Microbiol. 44, 103-118. doi: 10.1139/w97-135

Carnahan, A. M., Chakraborty, T., Fanning, G. R., Verma, D., Ali, A., Janda, J. M., et al. (1991). Aeromonas trota sp. nov., an ampicillin-susceptible species isolated from clinical specimens. J. Clin. Microbiol. 29, 1206-1210.

Chen, P. L., Tsai, P. J., Chen, C. S., Lu, Y. C., Chen, H. M., Lee, N. Y., et al. (2015). Aeromonas stool isolates from individuals with or without diarrhea in southern Taiwan: predominance of Aeromonas veronii. J. Microbiol. Immunol. Infect. 48, 618-624. doi: 10.1016/j.jmii.2014.08.007

Chu, Y. W., Wong, C. H., Tsang, G. K. L., Kwok, M. S. W., Wong, R. K. O., Lo, J. Y. C., et al. (2006). Lack of association between presentation of diarrhoeal symptoms and fecal isolation of Aeromonas spp. amongst outpatients in Hong Kong. J. Med. Microbiol. 55, 349-351. doi: 10.1099/jmm.0.46266-0

Crivelli, C., Demarta, A., and Peduzzi, R. (2001). Intestinal secretory immunoglobulin A ( $I$ IgA) response to Aeromonas exoproteins in patients with naturally acquired Aeromonas diarrhea. FEMS Immunol. Med. Microbiol. 30, 31-35. doi: 10.1111/j.1574-695X.2001.tb01546.x

Demarta, A., Tonolla, M., Caminada, A., Beretta, M., and Peduzzi, R. (2000). Epidemiological relationships between Aeromonas strains isolated from symptomatic children and household environments as determined by ribotyping. Eur. J. Epidemiol. 16, 447-453. doi: 10.1023/A:1007675424848

Denny, J. E., Powell, W. L., and Schmidt, N. W. (2016). Local and longdistance calling: conversations between the gut microbiota and intra- and extra-gastrointestinal tract infections. Front. Cell. Infect. Microbiol. 6:41. doi: $10.3389 /$ fcimb. 2016.00041 
DuPont, H. L., Hornick, R. B., Dawkins, A. T., Snyder, M. J., and Formal, S. B. (1969). The response of man to virulent Shigella flexneri 2a. J. Infect. Dis. 119, 296-299. doi: 10.1093/infdis/119.3.296

Egorov, A. I., Best, J. M., Frebis, C. P., and Karapondo, M. S. (2011). Occurrence of Aeromonas spp. in a random sample of drinking water distribution systems in the USA. J. Water Health. 9, 785-798. doi: 10.2166/wh.2011.169

Fernandez-Cassi, X., Silvera, C., Cervero-Aragó, S., Rusiñol, M., Latif-Eugeni, F., Bruguera-Casamada, C., et al. (2016). Evaluation of the microbiological quality of reclaimed water produced from a lagooning system. Environ. Sci. Pollut. Res. Int. doi: 10.1007/s11356-016-6812-0 [Epub ahead of print].

Figueras, M. J. (2005). Clinical relevance of Aeromonas. Rev. Med. Microbiol. 16, 145-153. doi: 10.1097/01.revmedmi.0000184410.98677.8a

Figueras, M. J., Aldea, M. J., Fernández, N., Aspíroz, C., Alperi, A., and Guarro, J. (2007a). Aeromonas hemolytic uremic syndrome. A case and a review of the literature. Diagn. Microbiol. Infect. Dis. 58, 231-234. doi: 10.1016/j.diagmicrobio.2006.11.023

Figueras, M. J., and Beaz-Hidalgo, R. (2014). “Aeromonas," in Encyclopedia of Food Microbiology, eds C. Batt and M. L. Tortorello (Oxford: Elsevier Ltd), 25-30.

Figueras, M. J., and Beaz-Hidalgo, R. (2015). “Aeromonas infections in humans," in Aeromonas, ed. J. Graf (Norfolk: Caister Academic Press), 65-108.

Figueras, M. J., Beaz-Hidalgo, R., Hossain, M. J., and Liles, M. R. (2014). Taxonomic affiliation of new genomes should be verified using average nucleotide identity and multilocus phylogenetic analysis. Genome Announc. 2: e927-14. doi: 10.1128/genomeA.00927-14

Figueras, M. J., and Borrego, J. J. (2010). New perspectives in monitoring drinking water microbial quality. Int. J. Environ. Res. Public Health 7, 4179-4202. doi: 10.3390/ijerph7124179

Figueras, M. J., Horneman, A. J., Martinez-Murcia, A., and Guarro, J. (2007b). Controversial data on the association of Aeromonas with diarrhoea in a recent Hong Kong study. J. Med. Microbiol. 56, 996-998. doi: 10.1099/jmm.0. 47062-0

Figueras, M. J., Suarez-Franquet, A., Chacón, M. R., Soler, L., Navarro, M., Alejandre, C., et al. (2005). First record of the rare species Aeromonas culicicola from a drinking water supply. Appl. Environ. Microbiol. 71, 538-541. doi: 10.1128/AEM.71.1.538-541.2005

Graf, J. (2015). Aeromonas. Norfolk: Caister Academic Press.

Granum, P. E., O'Sullivan, K., Tomas, J. M., and Ormen, O. (1998). Possible virulence factors of Aeromonas spp. from food and water. FEMS Immunol. Med. Microbiol. 21, 131-137. doi: 10.1111/j.1574-695X.1998.tb01158.x

Grim, C. J., Kozlova, E. V., Ponnusamy, D., Fitts, E. C., Sha, J., Kirtley, M. L., et al. (2014). Functional genomic characterization of virulence factors from necrotizing fasciitis-causing strains of Aeromonas hydrophila. Appl. Environ. Microbiol. 80, 4162-4183. doi: 10.1128/AEM.00486-14

Herrington, D., Tzipori, S., Robins-Browne, R., Tall, B., and Levine, M. (1987). In vitro and in vivo pathogenicity of Plesiomonas shigelloides. Infect. Immun. 55, 979-985.

Holmberg, S. D., Schell, W., Fanning, G., Wachsmuth, I., Hickman-Brenner, F., Blake, P., et al. (1986). Aeromonas intestinal infections in the United States. Ann. Int. Med. 105, 683-689. doi: 10.7326/0003-4819-105-5-690

Janda, J. M., and Abbott, S. L. (1998). Evolving concepts regarding the genus Aeromonas: an expanding panorama of species, disease presentation, and unanswered questions. Clin. Infect. Dis. 27, 332-344. doi: 10.1086/ 514652

Janda, J. M., and Abbott, S. L. (2010). The genus Aeromonas: taxonomy, pathogenicity, and infection. Clin. Microbiol. Rev. 23, 35-73. doi: 10.1128/CMR.00039-09

Katz, M. J., Parrish, N. M., Belani, A., and Shah, M. (2015). Recurrent Aeromonas bacteremia due to contaminated well water. Open Forum Infect. Dis. 2:ofv142. doi: 10.1093/ofid/ofv142

Khajanchi, B. K., Fadl, A. A., Borchardt, M. A., Berg, R. L., Horneman, A. J., Stemper, M. E., et al. (2010). Distribution of virulence factors and molecular fingerprinting of Aeromonas species isolates from water and clinical samples: suggestive evidence of water-to-human transmission. Appl. Environ. Microbiol. 76, 2313-2325. doi: 10.1128/AEM.02535-09

Krovacek, K., Dumontet, S., Eriksson, E., and Baloda, S. B. (1995). Isolation, and virulence profiles, of Aeromonas hydrophila implicated in an outbreak of food poisoning in Sweden. Microbiol. Immunol. 39, 655-661. doi: 10.1111/j.13480421.1995.tb03253.x
Latif-Eugenín, F. (2015). Aeromonas, Un Microorganismo Ambiental de Importancia en Salud Humana y Animal. Ph.D. thesis, Universitat Rovira I Virgili, Tarrogona. Available at: http://www.tdx.cat/ bitstream/handle/10803/334686/Tesi\%20Fadua.pdf?sequence=1\&isAllowed=y

Leggett, H. C., Cornwallis, C. K., and West, S. A. (2012). Mechanisms of pathogenesis, infective dose and virulence in human parasites. PLoS Pathog. 8:e1002512. doi: 10.1371/journal.ppat.1002512

Lund, B. M. (2015). Microbiological food safety for vulnerable people. Int. J. Environ. Res. Public Health 12, 10117-10132. doi: 10.3390/ijerph120810117

Lund, B. M., and O'Brien, S. J. (2011). The occurrence and prevention of foodborne disease in vulnerable people. Foodborne Pathog. Dis. 8, 961-973. doi: $10.1089 /$ fpd.2011.0860

Martin Delgado, M., Matute Cruz, P., Nuñez Gallo, D., Fernández González, C., López González Coviella, N., Valladares Hernández, B., et al. (2001). Diarrea del viajero asociada a Aeromonas hydrophila. Rev. Salud Ambient. 1, 30-35.

McDonald, S. A., Teunis, P., van der Maas, N., de Greef, S., de Melker, H., and Kretzschmar, M. (2015). An evidence synthesis approach to estimating the incidence of symptomatic pertussis infection in the Netherlands, 2005-2011. BMC Infect. Dis. 15:588. doi: 10.1186/s12879-0151324-y

McMahon, M. A., and Wilson, I. G. (2001). The occurrence of enteric pathogens and Aeromonas species in organic vegetables. Int. J. Food Microbiol. 70, 155162. doi: 10.1016/S0168-1605(01)00535-9

Morgan, D. R., Johnson, P. C., DuPont, H. L., Satterwhite, T. K., and Wood, L. V. (1985). Lack of correlation between known virulence properties of Aeromonas hydrophila and enteropathogenicity for humans. Infect. Immun. 50, 62-65.

Morinaga, Y., Yanagihara, K., Latif Eugenin, F. L., Beaz-Hidalgo, R., and Figueras, M. J. (2013). Identification errors of Aeromonas species: the considerable virulence of the new species Aeromonas aquariorum. Diag. Microbiol. Infect. Dis. 76, 106-109. doi: 10.1016/j.diagmicrobio.2013.01.019

Mosser, T., Talagrand-Reboul, E., Colston, S. M., Graf, J., Figueras, M. J., JumasBilak, E., et al. (2015). Exposure to pairs of Aeromonas strains enhances virulence in the Caenorhabditis elegans infection model. Front. Microbiol. 6:1218. doi: 10.3389/fmicb.2015.01218

Pablos, M., Huys, G., Cnockaert, M., Rodríguez-Calleja, J. M., Otero, A., Santos, J. A., et al. (2011). Identification and epidemiological relationships of Aeromonas isolates from patients with diarrhea, drinking water and foods. Int. J. Food Microbiol. 147, 203-210. doi: 10.1016/j.ijfoodmicro.2011. 04.006

Ponnusamy, D., Kozlova, E. V., Sha, J., Erova, T. E., Azar, S. R., Fitts, E. C., et al. (2016). Cross-talk among flesh-eating Aeromonas hydrophila strains in mixed infection leading to necrotizing fasciitis. Proc. Natl. Acad. Sci. U.S.A. 113, 722-727. doi: 10.1073/pnas.1523817113

Ramalivhana, J. N., Obi, C. L., Samie, A., Labuschagne, C., and Weldhagen, G. F. (2010). Random amplified polymorphic DNA typing of clinical and environmental Aeromonas hydrophila strains from Limpopo province, South Africa. J. Health Popul. Nutr. 28, 1-6.

Rendtorff, R. C. (1954). The experimental transmission of human intestinal protozoan parasites I. Giardia lamblia cysts given in capsules. Am. J. Hyg. 59, 209-220.

Ribet, D., and Cossart, P. (2015). How bacterial pathogens colonize their hosts and invade deeper tissues. Microbes Infect. 17, 173-183. doi: 10.1016/j.micinf.2015.01.004

Robertson, B. K., Harden, C., Selvaraju, S. B., Pradhan, S., and Yadav, J. S. (2014). Molecular detection, quantification and toxigenicity profiling of Aeromonas spp. in source and drinking water. Open Microbiol. J. 8, 32-39. doi: 10.2174/1874285801408010032

Senderovich, Y., Ken-Dror, S., Vainblat, I., Blau, D., Izhaki, I., and Halpern, M. (2012). A molecular study on the prevalence and virulence potential of Aeromonas spp. recovered from patients suffering from diarrhea in Israel. PLoS ONE 7:e30070. doi: 10.1371/journal.pone.0030070

Simonsen, J., Teunis, P., van Pelt, W., van Duynhoven, Y., Krogfelt, K., SadkowskaTodys, M., et al. (2011). Usefulness of seroconversion rates for comparing infection pressures between countries. Epidemiol. Infect. 139, 636-643. doi: 10.1017/S0950268810000750

Soler, L., Marco, F., Vila, J., Chacón, M. R., Guarro, J., and Figueras, M. J. (2003). Evaluation of two miniaturized systems, MicroScan W/A and BBL Crystal E/NF, 
for identification of clinical isolates of Aeromonas spp. J. Clin. Microbiol. 41, 5732-5734. doi: 10.1128/JCM.41.12.5732-5734.2003

Svenungsson, B., Lagergren, A., Ekwall, E., Evengård, B., Hedlund, K. O., Kärnell, A., et al. (2000). Enteropathogens in adult patients with diarrhea and healthy control subjects: a 1-year prospective study in a Swedish clinic for infectious diseases. Clin. Infect. Dis. 30, 770-778. doi: 10.1086/ 313770

Teunis, P., Falkenhorst, G., Ang, W., Strid, M., de Valk, H., SadkowskaTodys, M., et al. (2013). Campylobacter seroconversion rates in selected countries in the European Union. Epidemiol. Infect. 141, 2051-2057. doi: $10.1017 /$ S0950268812002774

Teunis, P., Kasuga, F., Fazil, A., Ogden, I., Rotariu, O., and Strachan, N. (2010). Dose response modeling of Salmonella using outbreak data. Int. J. Food Microbiol. 144, 243-249. doi: 10.1016/j.ijfoodmicro.2010. 09.026

Teunis, P., Ogden, I., and Strachan, N. (2008). Hierarchical dose response of E. coli O157:H7 from human outbreaks incorporating heterogeneity in exposure. Epidemiol. Infect. 136, 761-770. doi: 10.1017/S0950268807 008771

Teunis, P., Takumi, K., and Shinagawa, K. (2004). Dose response for infection by Escherichia coli O157:H7 from outbreak data. Risk Anal. 24, 401-407. doi: 10.1111/j.0272-4332.2004.00441.x

Teunis, P., van den Brandhof, W., Nauta, M., Wagenaar, J., van den Kerkhof, H., and van Pelt, W. (2005). A reconsideration of the Campylobacter dose-response relation. Epidemiol. Infect. 133, 583-592.

Teunis, P., van der Heijden, O. G., van der Giessen, J. W. B., and Havelaar, A. H. (1996). The Dose-Response Relation in Human Volunteers for GastroIntestinal Pathogens. Report nr. 284550002, 89. Bilthoven: Rijksinstituut voor Volksgezondheid en Milieu.

Teunis, P. F., Koningstein, M., Takumi, K., and van der Giessen, J. W. (2012a). Human beings are highly susceptible to low doses of Trichinella spp. Epidemiol. Infect. 140, 210-218. doi: 10.1017/S09502688110 00380

Teunis, P. F., van Eijkeren, J., Ang, W., van Duynhoven, Y., Simonsen, J., Strid, M., et al. (2012b). Biomarker dynamics: estimating infection rates from serological data. Stat. Med. 31, 2240-2248. doi: 10.1002/sim.5322
Teunis, P. F. M., and Havelaar, A. H. (2000). The Beta Poisson model is not a single hit model. Risk Anal. 20, 511-518. doi: 10.1111/0272-4332. 204048

Teunis, P. F. M., Nagelkerke, N. J. D., and Haas, C. N. (1999). Dose response models for infectious gastroenteritis. Risk Anal. 19, 1251-1260. doi: 10.1023/A:1007055316559

Thebault, A., Teunis, P., Le Pendu, J., Le Guyader, S., and Denis, J.-B. (2013). Infectivity of GI and GII noroviruses established from oyster related outbreaks. Epidemics 5, 98-110. doi: 10.1016/j.epidem.2012.12.004

Ventura, R. J., Muhi, E., de los Reyes, V. C., Sucaldito, M. N., and Tayag, E. (2015). A community-based gastroenteritis outbreak after Typhoon Haiyan, Leyte, Philippines, 2013. Western Pac. Surveill. Response J. 6, 1-6. doi: 10.2471/WPSAR.2014.5.1.010

Vila, J., Ruiz, J., Gallardo, F., Vargas, M., Soler, L., Figueras, M. J., et al. (2003). Aeromonas spp. and traveler's diarrhea: clinical features and antimicrobial resistance. Emerg. Infect. Dis. 9, 552-555. doi: 10.3201/eid0905.020451

von Graevenitz, A. (2007). The role of Aeromonas in diarrhea: a review. Infection 35, 59-64. doi: 10.1007/s15010-007-6243-4

Wadhwa, S. G., Khaled, G. H., and Edberg, S. C. (2012). Comparative microbial character of consumed food and drinking water. Crit. Rev. Microbiol. 28, 249-279. doi: 10.1080/1040-840291046740

Zhang, Q., Shi, G. Q., Tang, G. P., Zou, Z. T., Yao, G. H., and Zeng, G. (2012). A foodborne outbreak of Aeromonas hydrophila in a college, Xingyi City, Guizhou, China, 2012. Western Pac. Surveill. Response J. 3, 39-43. doi: 10.5365/WPSAR.2012.3.4.018

Conflict of Interest Statement: The authors declare that the research was conducted in the absence of any commercial or financial relationships that could be construed as a potential conflict of interest.

Copyright (๑) 2016 Teunis and Figueras. This is an open-access article distributed under the terms of the Creative Commons Attribution License (CC BY). The use, distribution or reproduction in other forums is permitted, provided the original author(s) or licensor are credited and that the original publication in this journal is cited, in accordance with accepted academic practice. No use, distribution or reproduction is permitted which does not comply with these terms. 